\title{
CoRad-19: Die radiologische Ausbildung geht digital weiter
}

Die Corona-Krise stellt auch Universitäten vor Probleme. Vorlesungen können derzeit nicht gehalten, Kurse nicht durchgeführt werden - eine Herausforderung für Dozentinnen und Dozenten wie Studierende, auch in der radiologischen Ausbildung. Die Deutsche Röntgengesellschaft e. V. (DRG) hat daher innerhalb kürzester Zeit ihre interaktive E-Learning-Plattform „conrad“ ausgebaut: Das dort zur Verfügung gestellte Angebot „unirad“ richtet sich mit seinen bereits bewährten Kursinhalten und -modulen an Medizinstudierende im Bereich Radiologie. Nun wurde unirad durch zahlreiche neue Kurse und Lehrmaterialen ergänzt - entstanden ist das modulare Kurssystem namens „CoRad-19“.

\section{Individuelles Lehrprogramm}

Basierend auf den bereits bestehenden E-Learning-Modulen des unirad-Angebots richtet sich das neue Notfallpaket CoRad-19 an Universitäten mit medizinischer Fakultät. Dabei kann jede Einrichtung entscheiden, welche CoRad-19-Kurse sie für die Lehre ein- setzen möchte, individuell auf die Bedürfnisse der eigenen Studentinnen und Studenten zugeschnitten. 13 Hochschulen von Lübeck über Tübingen bis Zürich nutzen das spezielle digitale Lehrangebot der DRG bereits. Nach einem absolvierten Online-Kurs erhält jeder Student beziehungsweise jede Studentin ein Zertifikat, welches er oder sie als Lernerfolgsnachweis einreichen kann.

\section{Interaktiv lernen und arbeiten wie in der Berufspraxis}

Für die Nutzung der CoRad-19-Kurse bedarf es keiner extra Software: Ein PC mit einer Internetverbindung reicht aus, um mittels der interaktiven fallbasierten Lehrmodule neues Wissen zu erwerben, es praxisnah anzuwenden und zu erweitern. Von Neuroradiologie über muskuloskelettale Radiologie bis hin zur interventionellen Radiologie decken die 10 Kurse aus dem CoRad-19-Angebot das gesamte Themenspektrum der radiologischen Ausbildung ab. Die Kursinhalte und Fallsammlungen wurden von 7 Expertinnen und Experten aufgearbeitet: Dr. Saif Afat (Tübingen), PD Dr. Bettina
Baeßler (Zürich), Dr. Nienke Hansen (Köln), Dr. Katharina Müller-Peltzer (Freiburg), PD Dr. Ahmed Othman (Tübingen), Dr. Daniel Pinto dos Santos (Köln) und Dr. Fabian Rengier (Heidelberg).

\section{Einbettung in erfolgreiches digitales Lehrkonzept}

CoRad-19 knüpft direkt an das bereits erfolgreich in conrad implementierte DRGAngebot für die digitale Lehre an. Bereits seit 2018 stehen mit dem unirad-Projekt umfangreiche und interaktive Kurse für Medizinstudierende bereit, die individuell an die jeweiligen universitätsmedizinischen Fakultäten angepasst werden können. Von der Online-PACS-Workstation und wichtigen Krankheitsbildern über fallbasierte MC-Fragen bis hin zu einem fallbasierten Nachschlagewerk: Die Inhalte sind didaktisch passend auf die Zielgruppe zugeschnitten. Jede Universität, die einen unirad-Kooperationsvertrag eingeht, erhält ab sofort auch automatisch Zugriff auf die ergänzenden modularen Lehrinhalte von CoRad-19. 


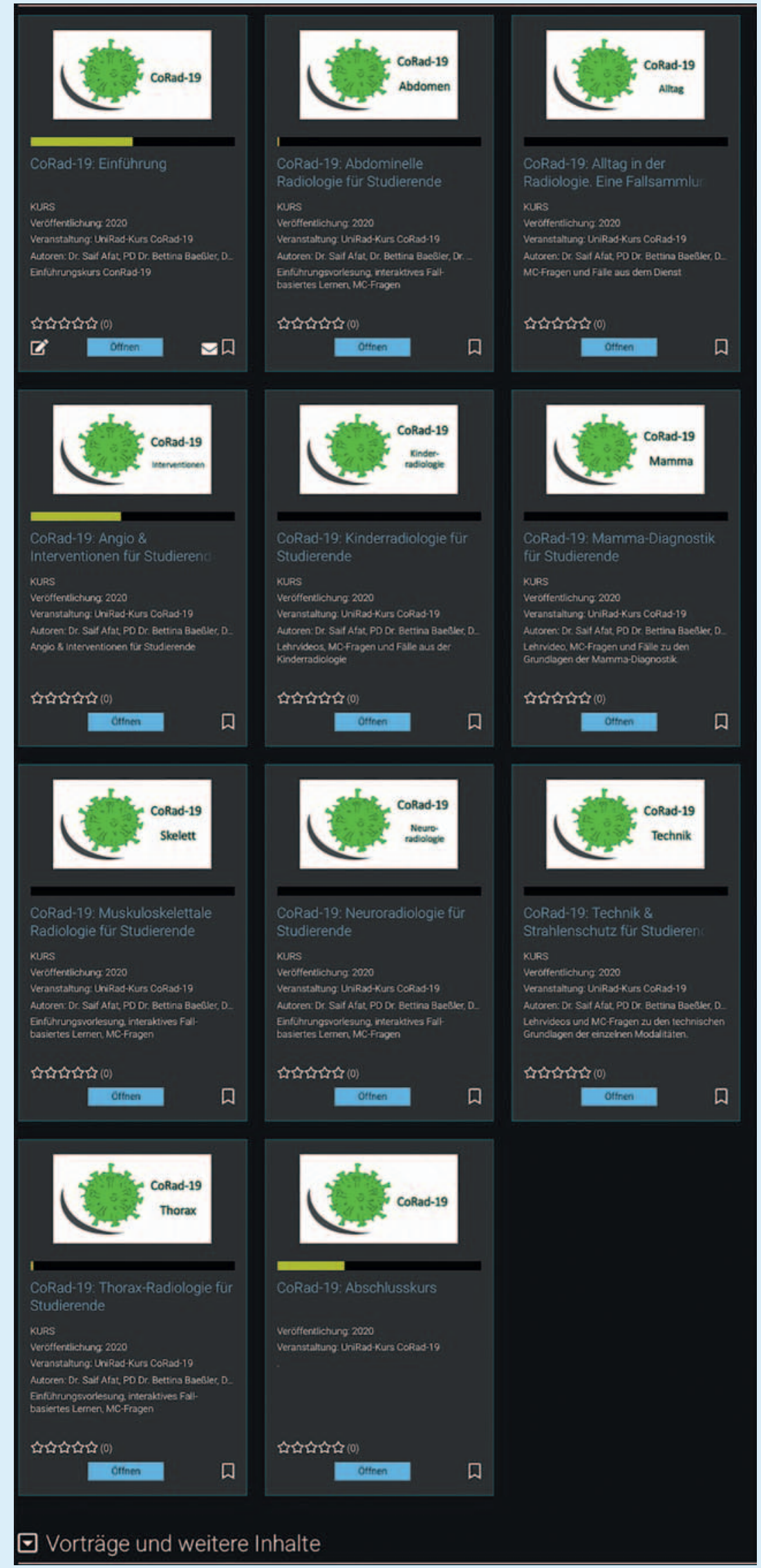

Überblick aller CoRad-19-Kurse @DRG 


\section{Die CoRad-19-Arbeitsgruppe}

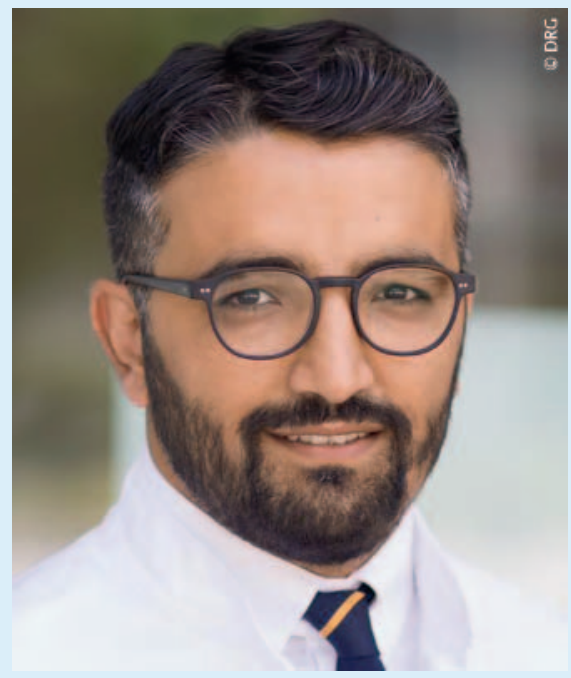

\section{Dr. Saif Afat}

„Das neue digitale Angebot deckt das radiologische Curriculum mit Vorlesungen und interaktiven Fallsammlungen nahezu vollständig ab. Somit bildet es in der derzeitigen Ausnahmesituation einen geeigneten Ersatz der Präsenzlehre."

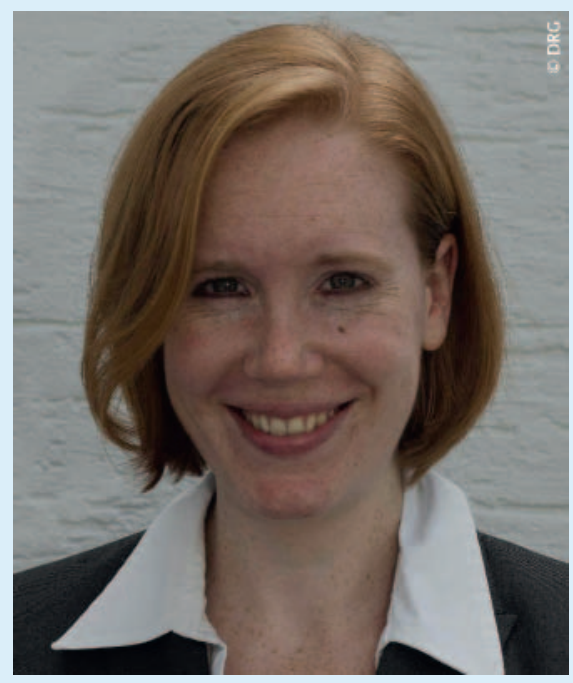

PD Dr. Bettina Baeßler

„Wenn die radiologischen Institute jetzt CoRad-19 in der Lehre benutzen, haben sie auch für „danach“ ein exzellentes Werkzeug an der Hand, wenn es um die Implementierung des neuen NKLM in Bezug auf die Bildgebung geht."

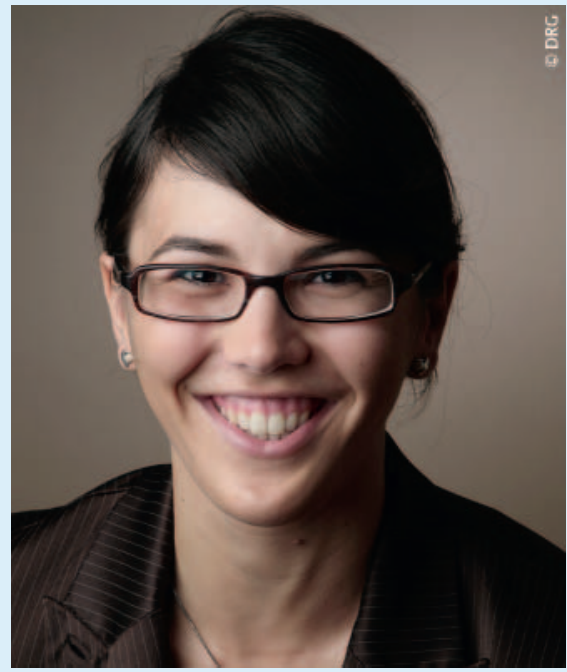

Dr. Nienke Hansen

„Im CoRad-19-Kurs üben die Studierenden selbstständig, die geeignete Untersuchungsmodalität oder das beste KM-Protokoll festzulegen sowie sich die wahrscheinlichste Differenzialdiagnose für einen Fall zu überlegen. Die Möglichkeit, durch den Bilddatensatz zu scrollen und die Fensterung zu optimieren, simuliert hierbei realitätsnah sowohl den Alltag in der Radiologie als auch die Arbeit im Nachtdienst in vielen weiteren Fachrichtungen. CoRad-19 kann die Präsenzlehre auch in Zukunft als Blended Learning optimal ergänzen, zum Beispiel im Rahmen eines Inverted-Classroom-Konzepts. “

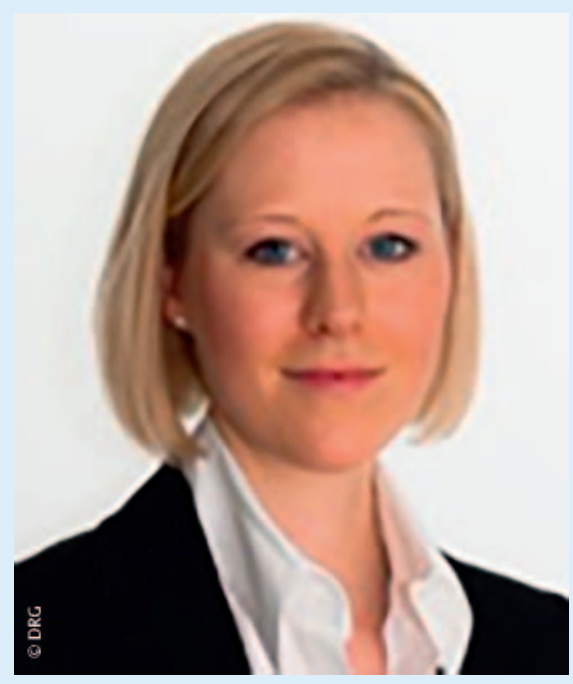

Dr. Katharina Müller-Peltzer

„CoRad-19 ist durch sein umfangreiches Curriculum, seine interaktive Lernumgebung sowie durch die fakultätsübergreifende Kooperation, aus der es hervorgegangen ist, ein gutes Beispiel für eine in der Krise genutzte Chance."

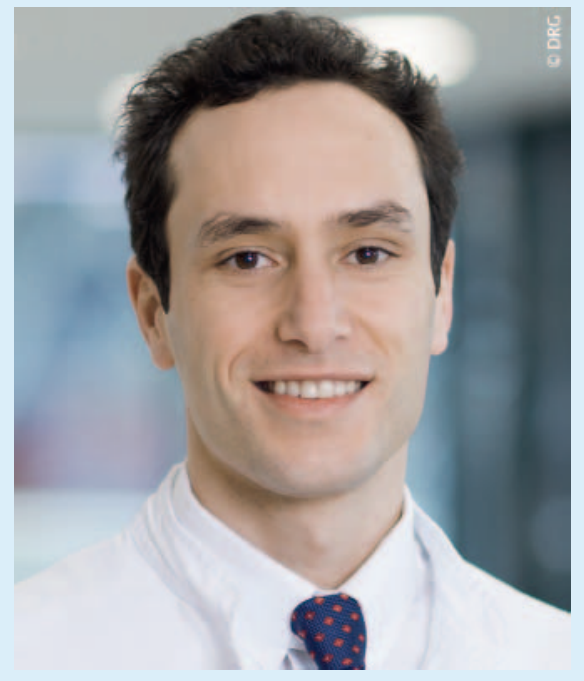

PD Dr. Ahmed Othman

„Die Studierenden können jederzeit und ortsunabhängig auf vollständige DICOM-Datensätze in unirad zugreifen. Eng orientiert an der Berufspraxis lernen sie, wie an einer „echten“ radiologischen PACS-Workstation zu arbeiten. “

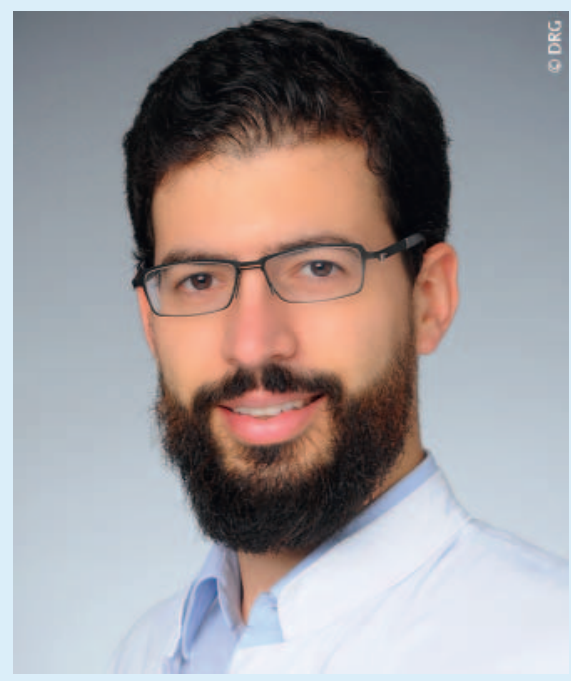

\section{Dr. Daniel Pinto dos Santos}

„Die CoRad-Initiative der DRG zeigt, dass wir innerhalb der Radiologie zu exzellenter Zusammenarbeit fähig sind und gemeinsam starke Lösungen für aktuelle Probleme finden können. Die studentische Lehre liegt uns sehr am Herzen und wir sind sicher, dass wir so allen Fakultäten qualitativ hochwertige Inhalte für eine digitale Lehre der Zukunft liefern können." 


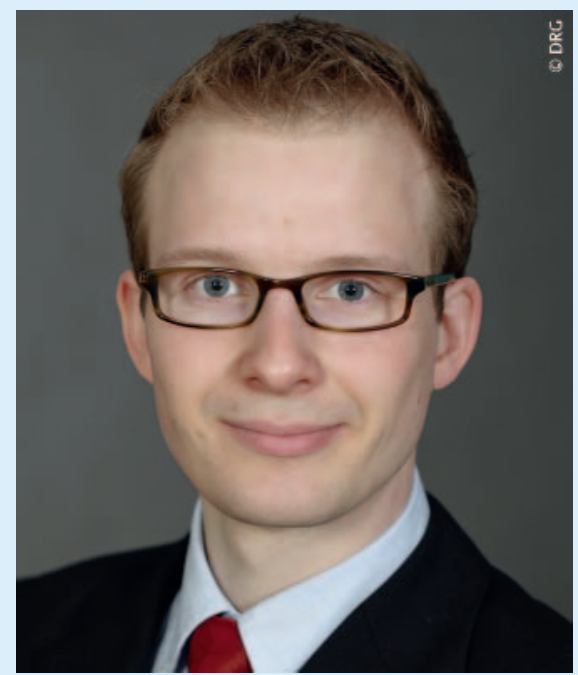

Dr. Fabian Rengier

„Die Einführung von unirad in unsere curriculare Lehre wurde von den Studierenden hervorragend evaluiert und resultierte in einer exzellenten Lernerfolgsquote. “

\section{INFORMATIONEN FÜR UNIVERSITÄTEN}

Deutsche Röntgengesellschaft e. V.

Olaf Goldschmidt

Ernst-Reuter-Platz 10

10587 Berlin

Tel.: + 49 (0)3091607047

E-Mail: goldschmidt@drg.de

www.drg.de 